\title{
STEM-tomography in SEM
}

Luyang Han ${ }^{1}$, Markus Boese ${ }^{1}$, Benjamin Tordoff ${ }^{2}$, Matthew Andrew ${ }^{3}$ and Evan Drake ${ }^{3}$

${ }^{1}$ Carl Zeiss Microscopy GmbH, Oberkochen, Germany, ${ }^{2}$ Carl Zeiss Microscopy GmbH, Germany, ${ }^{3}$ arl Zeiss X-ray Microscopy, United States

3D information at the highest resolution can be achieved by using nano tomography. Common approaches are FIB/SEM tomography with subsequent slicing of the sample or using tilt series in transmission by (S)TEM tomography. (S)TEM tomography is usually achieved by either bright field transmission electron microscopy (BF-TEM) or annular dark-field scanning transmission electron microscopy (ADF-STEM), both of which are usually performed in a TEM setup with high electron beam energy. Normal scanning electron microscope equipped with a dedicated detector gives the possibility to perform STEM imaging, though at a much lower energy level and lower resolution. Standard SEM instrumentation is not designed for tomography: the limited tilt range of the stage and detector geometry commonly prohibits imaging with large tilt. To overcome such limitations researchers have constructed customized stage and detector systems, demonstrating the feasibility of ADF-STEM tomography in SEM [1]. In this contribution we report the realization of STEM tilt series and tomography in a normal SEM setup using a specially designed sample holder but with the standard sample stage and STEM detector.

The configuration of the system is shown in Figure 1. The sample holder encompasses cross shaped cantilevers which hold the samples on the edges. The sample holder is then mounted on the standard SEM stage. The sample is placed into the gap between the STEM detector and the objective lens. The whole system can be tilted up to $60^{\circ}$ at a working distance of $4 \mathrm{~mm}$, while maintaining a safe distance to the objective lens and the detector. As a standard SEM stage can only tilt to large angles in a positive direction, an equivalent negative tilt is achieved by rotating the sample $180^{\circ}$ around the STEM detector and then tilt in the positive direction.

A major problem when using a normal SEM system to capture high resolution STEM tilt series is the limited stage accuracy. To keep track of the region of interest compu-centric rotation and tilt is used, but this gives only an accuracy in 10s of micrometers, while typical STEM images are captured with field of view in just a few micrometers. To overcome such limitation a robust feature tracking routine is developed to search and center the region of interest at gradually increasing magnification using successive stage movement and beam shift. The final tilt series show a residue image drifts less than $60 \mathrm{~nm}$. Such feature tracking routine is integrated in a computer program developed to automate the acquisition the API interface of the SEM.

Figure 2 shows an exemplary result of such tilt series. The investigated sample is $\mathrm{ZnO}$ nanoparticles dispersed on carbon grid. The sample is imaged at $30 \mathrm{kV}$ beam energy with tilt angle from $-55^{\circ}$ to $55^{\circ}$. The tilt image series are then aligned and reconstructed using filtered back projection. Although the result still contains significant limited angle artifacts, the 3D shapes of the particles can be well resolved. 


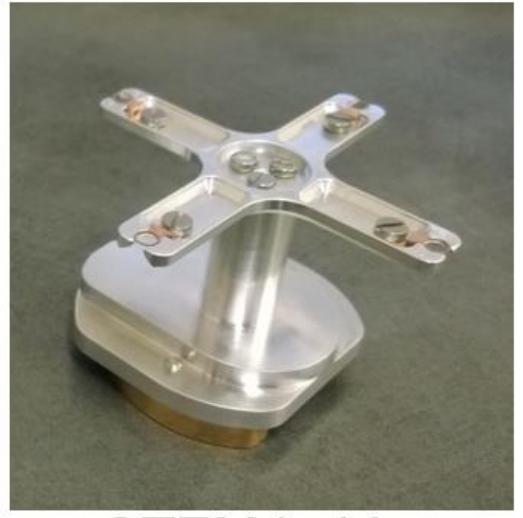

STEM holder
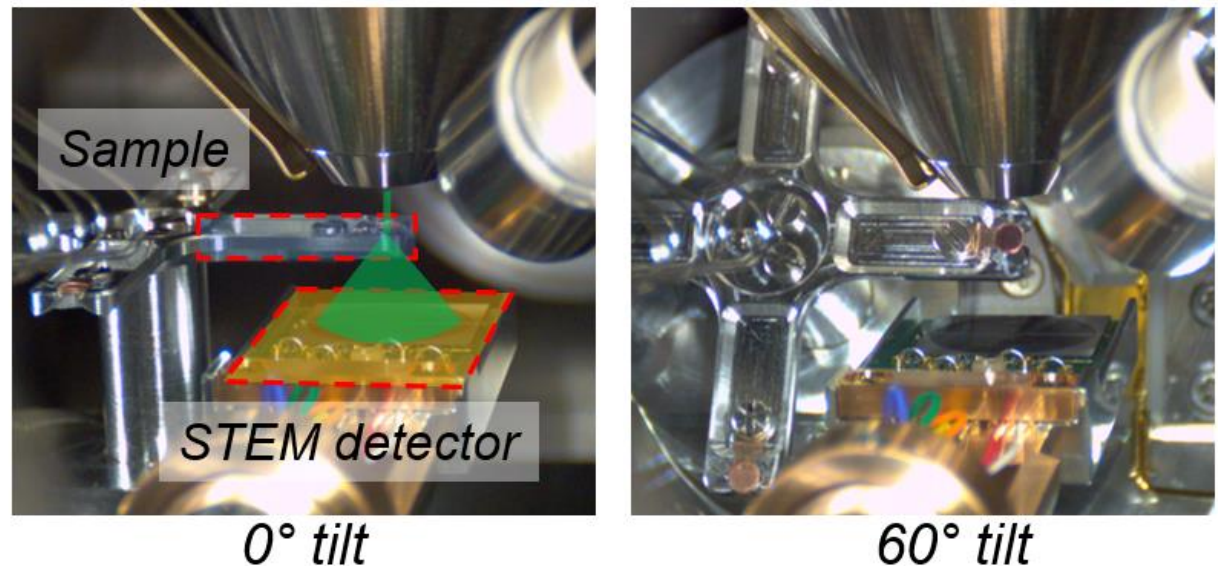

Figure 1. Configuration of STEM tomography in SEM. The left image shows the dedicated STEM tomography holder. The holder is placed between the objective lens and STEM detector shown in the middle. The right image shows the situation at $60^{\circ}$ tilt.
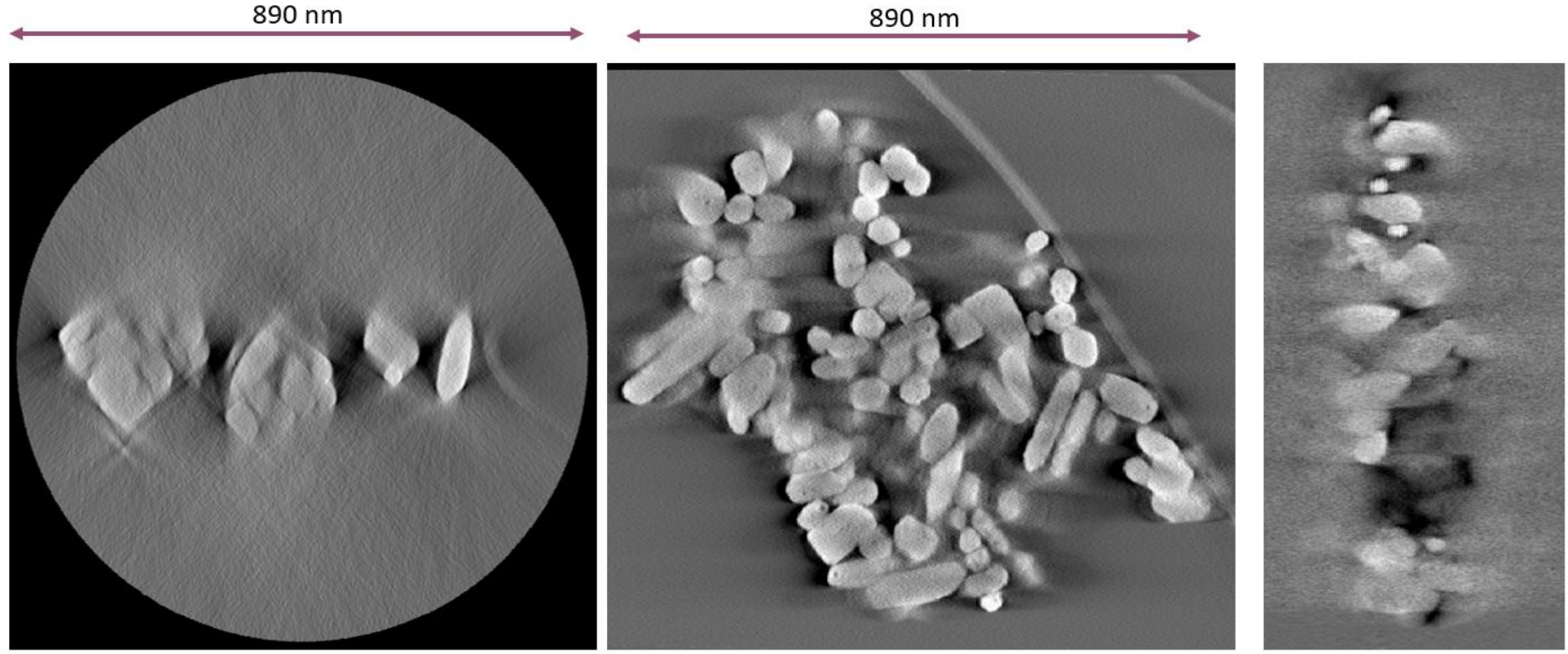

Figure 2. Tomography reconstruction of tilt series of $\mathrm{ZnO}$ nanoparticles shown as the cross-section in $\mathrm{X}$, $\mathrm{Y}$ and $\mathrm{Z}$ planes. The limited angle artifacts can be seen in the reconstruction.

References

[1] V. Morandi et al, AIP Conference Proceedings 1667, 020013 (2015) 